\title{
Glottopol
}

Revue de sociolinguistique en ligne

$34 \mid 2020$

Les « langues de France ", 20 ans après

\section{Entretien avec M. Paul de Sinety, délégué général à la langue française et aux langues de France}

\section{Christian Lagarde}

\section{(2) OpenEdition \\ Journals}

Édition électronique

URL : https://journals.openedition.org/glottopol/355

DOI : 10.4000/glottopol.355

ISSN : 1769-7425

Éditeur

Presses universitaires de Rouen et du Havre

\section{Référence électronique}

Christian Lagarde, « Entretien avec M. Paul de Sinety, délégué général à la langue française et aux langues de France », Glottopol [En ligne], 34 | 2020, mis en ligne le 01 juillet 2020, consulté le 02 octobre 2021. URL : http://journals.openedition.org/glottopol/355 ; DOI : https://doi.org/10.4000/ glottopol.355 


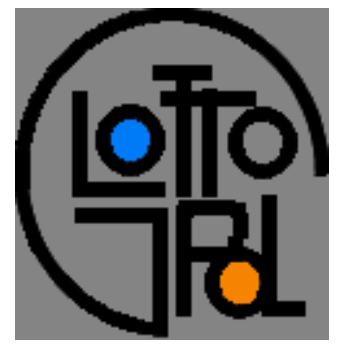

\section{GLOTTOPOL}

Revue de sociolinguistique en ligne $\mathrm{n}^{\circ} 34$ - juillet 2020

Les "langues de France " : 20 ans après

\section{SOMMAIRE}

Hommages à Jean Le Dî

Christian Lagarde : "Langues de France ». Au-delà du symbolique?

Entretien avec Bernard Cerquiglini, par Christian Lagarde.

Entretien avec Paul de Sinety, Délégué général à la langue française et aux langues de France, par Christian Lagarde.

Georg Kremnitz : La problématique initiale de la liste Cerquiglini et ses effets ultérieurs.

Alain Viaut : De "langue régionale » à "langue de France » ou les ombres du territoire.

Wanda Mastor: Le statut constitutionnel des langues régionales en droit comparé. De la reconnaissance à l'indifférence.

Philippe Martel, Marie-Jeanne Verny: Les langues régionales au Parlement, ou l'éternel retour.

Romain Colonna: Les «langues de France» : des langues non-étatiques au pays de l'Étatnation.

Hervé le Bihan : La langue bretonne : une visibilité toute en retenue.

Véronique Bertile : Les langues d'outre-mer : des langues de France? Approche juridique.

Jacques Vernaudon: Les langues polynésiennes et kanak, des "langues de France» en contexte de décolonisation.

Luc Biichlé : Qu'advient-il de l'arabe de France? Mise en perspective sociolinguistique...

Pascal Ottavi : L'épervier, la cage et le passereau.

Marielle Rispail : Le francique lorrain, langue de France? Réflexions et témoignages.

\section{Compte rendu de lecture}

Par Salih Akin : Jean Le Dû \& Yves Le Berre, Métamorphoses. Trente ans de sociolinguistique à Brest (1984-2014), Brest, Centre de Recherche Bretonne, 2019, 302 p.

http://glottopol.univ-rouen.fr 


\title{
ENTRETIEN AVEC M. PAUL DE SINETY, DÉLÉGUÉ GÉNÉRAL À LA LANGUE FRANÇAISE ET AUX LANGUES DE FRANCE
}

\author{
Par Christian Lagarde
}

\section{Quelques repères}

C'est en vue de la signature de la Charte européenne des langues régionales ou minoritaires qu'a été élaboré en 1999 - il y a environ vingt ans - le dénommé « Rapport Cerquiglini », du nom de son auteur, alors directeur de l'INALF (Institut national de la langue française). On sait que c'est afin que la France soit en mesure de satisfaire à la sélection de critères imposée par la Charte qu'a ainsi émergé, au-delà de la traditionnelle appellation "langues régionales", celle de "langues de France». Il aura donc fallu une stimulation externe pour que l'État, qui s'inscrivait dans la perspective inverse, de la mention restrictive "Le français est la langue de la République », en 1992, à la Loi Toubon (1994), dont les effets sont somme toute semblables à ceux de l'Ordonnance de Villers-Cotterêts de 1539 (celle-ci dirigée à l'encontre du latin, celle-là, de l'anglais), se préoccupe de se pencher sur sa diversité linguistique interne...

L'adoption de la Charte européenne des langues régionales et minoritaires par le Conseil de l'Europe et l'inscription dans la constitution française «La langue de la République est le français » ont eu lieu le même jour, le 25 juin 1992. Il s'agit là d'une coïncidence frappante. La France a sans aucun doute perçu comme une nécessité de rééquilibrer sa politique linguistique en affirmant par sa Constitution l'indivisibilité de la République par la langue française. La loi Toubon a confirmé cette disposition, même si elle avait pour objectif principal de faire pièce à l'emploi abusif de termes étrangers (essentiellement anglais), dans une optique de protection du citoyen et du consommateur. Ce qui est certain, c'est que la loi Toubon n'avait pas, en principe, vocation à s'opposer à l'usage des langues régionales, comme l'exprime clairement son article 21 : «les mesures garantissant l'emploi de la langue française s'appliquent sans préjudice de la législation et de la réglementation relatives aux langues régionales de France et ne s'opposent pas à leur usage ». Il est vrai que cet article est régulièrement ignoré des juridictions administratives...

Cela dit, la France n'a pas attendu « une stimulation externe » pour prendre en compte sa diversité linguistique. La loi Deixonne relative à l'enseignement des langues et dialectes locaux date de 1951. Certes elle ne concernait à l'origine que quatre langues (basque, breton, catalan et occitan), mais c'était déjà une première reconnaissance. 


\section{Sens et contours de « langues de France»}

Au-delà de l'innovation terminologique, la conception même de "langues de France 》, si j'ose dire, rebat les cartes. D'une part en affirmant ainsi leur appartenance, en quelque sorte indissoluble, à la nation et à l'État (qui en France, sont censés se confondre), d'autre part, en incluant d'autres catégories de langues. Certains ont de ce fait considéré la liste des 75 " langues de France » comme un « inventaire à la Prévert». Qu'en pensez-vous?

Il n'est pas tout à fait exact de parler d'innovation terminologique. L'expression « langues de France » est attestée dès 1905, on la trouve chez Antonin Perbosc, instituteur qui avait adopté les méthodes d'enseignement dites « actives », et qui écrivait en tant que pédagogue des langues de France.

Le rapport Cerquiglini comportait 75 glossonymes. Cette liste n'était en rien figée comme on l'a vu par la suite puisque d'autres langues sont venues l'enrichir : le champenois, les parlers liguriens, le judéo-espagnol ou ladino, la langue des signes française (LSF). Certaines langues des Outre-mer ont été renommées au fil des années... D'autres langues en sont absentes et mériteraient sans doute d'y être incluses : les parlers du Croissant, le créole anglais de SaintMartin. Quoi qu'il en soit, cette liste n'est en aucun cas un inventaire à la Prévert, elle reflète au contraire la réalité linguistique de notre pays. La France est le pays d'Europe qui connait la plus grande diversité linguistique, notamment grâce aux territoires ultra-marins : rien qu'en Nouvelle-Calédonie, on ne compte pas moins de 28 langues !

C'est vrai que le rapport Cerquiglini a été élaboré dans l'objectif de la Charte : il s'agissait de lister les langues qui étaient concernées par les dispositions de la Charte. Or, la Charte exclut les langues issues de l'immigration et par conséquent certaines de nos langues non territoriales ne sont a priori pas concernées (arabe dialectal, berbère, arménien occidental...).

La décision d'intégrer dans la liste des langues de France des langues qualifiées de «nonterritoriales » bien que solidement territorialisées à l'étranger - et au rebours de l'esprit de la Charte européenne - devrait sans doute être reconsidérée. La responsabilité que le pays se reconnait vis-à-vis de ces langues peut sans doute s'exprimer d'une autre manière, dans un autre cadre : ce qui se produit, d'ailleurs, à l'Inalco, à l'Université, etc.

Je comprends que cette liste ait pu étonner nombre de concitoyens, mais je crois qu'il faut s'en réjouir. Les Français ont découvert cette richesse linguistique, ce qui rompt avec l'ignorance et le désintérêt qui avaient prévalu jusqu'alors. Cette richesse linguistique a été trop longtemps occultée voire niée.

Prenons un cas particulier, singulièrement problématique à bien des égards au regard de la société française contemporaine: la langue arabe est considérée ici à travers les seuls dialectes présents sur d'anciens territoires coloniaux, et non pas en tant que langue étrangère ou d'immigration. Au-delà de la portée symbolique (répondant sans doute, dans l'apaisement, à la "violence symbolique" exercée par le dominantlle pouvoir, mise en évidence par Bourdieu), par quels effets pratiques s'est traduite depuis lors une telle inclusion au sein des "langues de France»?

L'inclusion de l'arabe dialectal sur la liste des langues de France n'a eu aucune incidence particulière pour cette langue. L'arabe dialectal n'est pas enseigné en France (ou alors de façon très marginale et en dehors de l'éducation nationale), il reste une langue orale qui continue d'être transmise sous ses formes algérienne, marocaine ou tunisienne.

L'arabe dialectal a été reconnu comme langue de France car il répondait aux critères des langues non territoriales : langue issue de l'immigration, pratiquée par des Français depuis plusieurs générations, sans lien avec une aire géographique de notre territoire, sans caractère 
officiel dans les pays d'où elle est originaire (la langue officielle de l'Algérie, du Maroc ou de la Tunisie est l'arabe littéral). En 2009, notre revue Langues et Cité a consacré un numéro à l'arabe, présenté comme un continuum : dialectal (dans l'espace), mais aussi dans le temps. Il s'agit d'une représentation unitaire à laquelle les locuteurs sont partout très attachés. Cela affaiblit sans doute la distinction entre l'arabe classique-officiel et l'arabe dialectal, sur laquelle repose la présence du dialectal dans la liste.

Le berbère était dans la même situation que l'arabe dialectal jusqu'à ce qu'il devienne langue officielle au Maroc en 2011 et en Algérie en 2016. En raison de cette officialisation, il ne répond plus complètement à la définition d'une langue de France non territoriale.

D'une manière générale, je crois qu'il faut poursuivre notre réflexion autour de la liste des langues de France, ce qui est un exercice très délicat : attribution du statut de langue ou de dialecte, choix de regrouper certaines langues, etc. Ces questions ont déjà été soulevées par le rapport du Comité consultatif pour la promotion des langues régionales et de la pluralité linguistique interne «Redéfinir une politique en faveur des langues régionales et de la pluralité linguistique interne », remis à Aurélie Filippetti en 2013.

\section{La « marque » Cerquiglini}

Le Rapport Cerquiglini peut être regardé, avec la distance temporelle, comme un texte de circonstance. Après sa signature, la ratification de la Charte est restée 'à quai', si vous me permettez l'expression...

La Charte a été signée le 7 mai 1999 mais n'a pas été ratifiée suite à l'avis négatif du Conseil constitutionnel. Deux autres tentatives de la faire ratifier n'ont pu aboutir, en 2008 et en 2015. Pour autant, il ne faut pas faire une fixation sur cette non-ratification. En signant la Charte, la France a pris 39 engagements, au-delà des 35 minimums requis, dans les domaines les plus divers : enseignement, culture et médias, vie sociale et économique, services publics. La plupart de ces engagements étaient déjà appliqués, la signature n'a fait qu'officialiser des pratiques qui avaient déjà cours (notamment l'enseignement des langues régionales et leur présence dans l'espace public à travers la signalisation bilingue dans certaines régions, etc.). Il reste encore beaucoup à faire pour donner aux langues régionales toute la place qu'elles méritent, notamment dans les services publics et dans l'éducation, mais il serait faux de prétendre que l'État n'a rien fait en leur faveur. C'est vrai que pendant longtemps, la France a refusé de se voir dans un miroir et a entretenu le mythe d'un pays monolingue, ce qu'elle n'a jamais été : jusqu'en 1914, la langue première de la majorité des Français n'était pas le français.

Un autre apport, sans doute lui aussi considérable, dont nous sommes redevables à Bernard Cerquiglini, est le passage - en bonne logique de son action - de la Délégation générale à la langue française, à celle-là même, accompagnée de "et aux langues de France ». Qu'est-ce que cela a changé dans les missions de l'ex-DGLF? Peut-on considérer cette évolution d'un point de vue idéologique, en matière de politique linguistique de la France? Au-delà d'une évolution dans l'organigramme, comment s'est traduite, concrètement, au plan des interventions, cette inflexion en faveur du pluri- / multilinguisme tel qu'il existe de fait sur le territoire français? Quel bilan peut-on aujourd'hui tracer, du point de vue de l'institution, de ces vingt années?...

C'est en 2001 que la DGLF est devenue DGLFLF, par décret ministériel. La nomination de Bernard Cerquiglini en tant que délégué général a coïncidé avec ce changement. Cela dit, dans les années précédant la signature de la Charte, la DGLF avait déjà étendu ses missions à la 
diversité linguistique interne. Dès les années 1980, la Direction du développement culturel du Ministère de la Culture avait une Mission des cultures régionales et communautaires, qui incluait la dimension linguistique.

Le changement en DGLFLF a eu des répercussions importantes dans notre politique linguistique. Tout d'abord, pour la première fois de son histoire l'État introduisait la mention « langues de France » dans l'un de ses services. Il est vrai que la position de la France était devenue intenable sur la scène internationale dans le sens où elle ne pouvait pas continuer de prôner le multilinguisme sans tenir compte de sa réalité linguistique interne. Dès 1999, la DGLF s'était dotée d'un Observatoire des pratiques linguistiques pour conduire une politique s'appuyant sur des études scientifiques. Dès 2001, la DGLFLF a pris des mesures favorables aux langues de France : subventions accordées à la recherche et aux projets culturels en langues régionales. Au fil des ans, elle a apporté un soutien considérable aux diverses structures opérant en faveur des langues régionales (les offices publics des langues basque, bretonne, occitane, catalane, le CIRDOC, etc.), mais aussi au tissu associatif. Elle a aussi développé des outils numériques pour permettre à ces langues d'entrer de plain-pied dans la modernité du $21^{\mathrm{e}}$ siècle. Il est très important de casser les préjugés négatifs, l'image passéiste et folklorique dont sont souvent victimes les langues régionales. Il n'y a pas de hiérarchie scientifique entre les langues, elles sont toutes capables d'exprimer le monde dans sa complexité, seuls les outils utilisés sont différents.

En 2003, la DGLFLF a organisé un événement tout à fait inédit: les premières Assises nationales des langues de France. Lors de son allocution d'ouverture, Jean-Jacques Aillagon, alors ministre de la Culture, avait prononcé quelques phrases en francique mosellan qui était la langue maternelle de sa mère. Qu'un ministre du gouvernement français se soit exprimé publiquement dans une langue régionale a été symboliquement très fort.

Quelques années plus tard, en 2011, la DGLFLF a organisé les premiers États généraux du multilinguisme dans les Outre-mer, à Cayenne. La situation linguistique des Outre-mer n'est pas la même qu'en métropole, et cette différence doit être prise en compte dans les politiques linguistiques. Dans les Outre-mer, le français est loin d'être partout la langue première de nos compatriotes. En 2021, la seconde édition des États généraux du multilinguisme dans les Outremer aura lieu à Saint-Denis de la Réunion. Il s'agira de dresser un bilan du chemin parcouru en dix ans, depuis la Déclaration de Cayenne, et de poursuivre une politique d'ouverture prenant pleinement en compte la spécificité linguistique des Outre-mer.

Plus précisément, quelle influence la DGLFLF peut-elle avoir en matière de politique linguistique, dans des domaines tels que la culture, l'enseignement, les médias? La dernière discussion parlementaire sur les langues régionales est celle du projet de loi $n^{\circ} 4096^{1}$. Elle a à nouveau débouché sur une impasse, mis en évidence les blocages, pour la plupart idéologiques, qui parcourent, au-delà même des clivages partisans, la représentation nationale. Quelles stratégies peut, dès lors, adopter et mettre en ouvre la DGLFLF afin de "faire bouger les choses »?... En a-t-elle véritablement les moyens, sous la présidence Macron et le gouvernement actuel?

Sur les points culturels, je vous renvoie à la question précédente. La question de l'enseignement relève directement du Ministère de l'Éducation nationale. Le Code de l'éducation prévoit sans ambiguiité l'enseignement d'une bonne quinzaine de langues et cultures régionales. Dans les Outre-mer, des approches spécifiques sont prévues pour les enfants scolarisés dans le premier degré et qui n'ont pas le français pour langue première. Mais

\footnotetext{
${ }^{1}$ À la date de réalisation de cet entretien, la Proposition de loi no 321 relative à la protection patrimoniale des langues régionales et à leur promotion, déposée le vendredi 14 février 2020, par le député breton Paul Molac, adoptée en première lecture par l'Assemblée nationale, n'était pas encore d'actualité.
} 
beaucoup reste sans doute à faire, notamment au niveau du collège et du lycée. Les réformes du lycée et du baccalauréat mises en place cette année suscitent l'inquiétude de nombreux professeurs et parents d'élèves.

Pour les médias, il y a encore des progrès à faire tant la présence des langues régionales est inégalement répartie selon les langues et les régions.

\section{L'article 75-1 : une déconvenue ? Et au-delà ?...}

En 2008, la Constitution - sans doute pour remédier à la fermeture que suppose la réduction unilingue introduite par la mention nouvelle apportée en 1992 à l'article 2 de la Constitution - se voit compléter par l'article 75-1, qui reconnait la dimension patrimoniale des langues régionales. Ce n'est là, de mon point de vue, qu'une maigre compensation. La visée patrimoniale est par essence rétrospective, et non prospective, un projet. Le "plébiscite de tous les jours " de la nation - pour convoquer Renan - se garderait-il bien d'ouvrir la boite de Pandore des aspirations centrifuges qui se manifestent ici ou là? Quel est donc selon vous le devenir qui est ainsi tracé pour ces langues? Quelles actions concrètes sont envisageables dans cette perspective?

Avec l'article 75-1, la reconnaissance des langues régionales est inscrite noir sur blanc dans la Constitution. La France n'est plus dans une posture de déni, elle accepte et valorise aujourd'hui sa diversité linguistique. On peut néanmoins déplorer que la seule dimension patrimoniale ait été retenue pour caractériser les langues régionales. Quoi qu'en disent leurs détracteurs, les langues régionales de France sont vivantes, elles sont parlées quotidiennement, elles s'adaptent tant bien que mal à leur situation de langues minoritaires, ce qui ne les empêche pas d'être dynamiques et créatives. Il serait sans doute plus pertinent de parler de ressources linguistiques qui peuvent être exploitées.

Les aspirations centrifuges que vous mentionnez sont des préjugés sans fondement, elles n'existent pas. Les locuteurs de langues régionales peuvent parfaitement se sentir français sans pour autant renier une langue qui les définit au même titre, voire dans certains cas davantage, que la langue française.

Au-delà, comment interpréter le fait que, 9 ans après le Rapport, 7 ans après l'addendum LFLF, l'article 75-1 concerne les "langues régionales 》 et non les "langues de France 》? Comment considérer le fait que, au-delà de leur dimension circonstancielle puis institutionnelle, elles n'aient pas acquis une dimension légale - qui plus est au sein du texte juridique de plus haut niveau de la République française? La DGLFLF ne se trouve-t-elle pas dès lors dans une sorte de porte-à-faux? Et les "langues de France" sur une "voie de garage $» ? . .$.

Je ne pense pas qu'il faille considérer les choses sous cet angle. Certes, le concept de « langues régionales » est plus étroit puisqu'il exclut les langues non territoriales, issues de l'immigration (arabe dialectal, arménien occidental, berbère, judéo-espagnol, romani, yiddish). Que les langues non territoriales soient inscrites ou non dans la Constitution ne change rien à leur statut, elles restent des langues de France, tout comme la langue des signes qui est une langue non territoriale mais sans rapport avec l'immigration. Depuis 2005, elle est reconnue comme une langue à part entière (article 75 de la Loi pour l'égalité des droits et des chances, la participation et la citoyenneté des personnes handicapées). 
Pour conclure, à travers ce que l'on peut en percevoir du point de vue de l'institution officielle que vous représentez, y a-t-il une véritable volonté politique de sauvegarder les 75 ou 78 «langues de France »? Et si oui, dans quelles limites?...

Bien sûr. Il en va du destin des 78 langues de France comme du destin du français. Le français est aussi une langue de France, c'est une langue d'oïl qui a eu la chance d'être jadis la langue du roi. Il est très important aujourd'hui de promouvoir le multilinguisme non seulement en France, mais aussi à l'échelle mondiale. 


\section{GLOTTOPOL}

Revue de sociolinguistique en ligne

Comité de rédaction : Michaël Abecassis, Salih Akin, Sophie Babault, Claude Caitucoli, Véronique Castellotti, Régine Delamotte, Robert Fournier, Stéphanie Galligani, Emmanuelle Huver, Normand Labrie, Foued Laroussi, Benoit Leblanc, Fabienne Leconte, Gudrun Ledegen, Danièle Moore, Clara Mortamet, Alioune Ndao, Isabelle Pierozak, Gisèle Prignitz.

Rédactrice en chef : Clara Mortamet.

Comité scientifique : Claudine Bavoux, Michel Beniamino, Jacqueline Billiez, Philippe Blanchet, Pierre Bouchard, Ahmed Boukous, Pierre Dumont, Jean-Michel Eloy, Françoise Gadet, Monica Heller, Caroline Juilliard, Jean-Marie Klinkenberg, Jean Le Dû $(\dagger)$, Marinette Matthey, Jacques Maurais, Marie-Louise Moreau, Robert Nicolaï, Didier de Robillard, Paul Siblot, Claude Truchot, Daniel Véronique.

\section{Comité de lecture pour ce numéro :}

Salih Akin, Carmen Alén Garabato, Sophie Babault, Philippe Blanchet, Henri Boyer, Véronique Castellotti, Marisa Cavalli, Jean-François De Pietro, Didier de Robillard, Alain Di Meglio, Ksenija Djordjevic, Jean Michel Eloy, Pascale Erahrt, Véronique Fillol, Monica Heller, Robert Fournier, Normand Labrie, Hervé Lieutard, Jean Le Dû (†), Marinette Matthey.

http://glottopol.univ-rouen.fr

ISSN : 1769-7425 